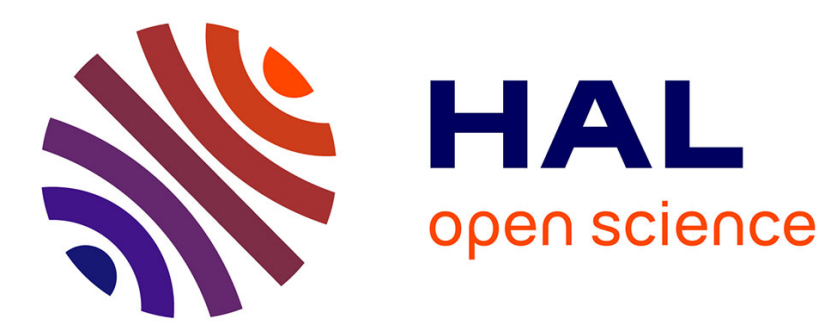

\title{
Separation of Sparse Signals in Overdetermined Linear-Quadratic Mixtures
}

Leonardo Tomazeli Duarte, Rafael Assato Ando, Romis Attux, Yannick Deville, Christian Jutten

\section{- To cite this version:}

Leonardo Tomazeli Duarte, Rafael Assato Ando, Romis Attux, Yannick Deville, Christian Jutten. Separation of Sparse Signals in Overdetermined Linear-Quadratic Mixtures. LVA/ICA 2012 - 10th International Conference on Latent Variable Analysis and Signal Separation, Mar 2012, Tel-Aviv, Israel. pp.239-246. hal-00687408

\section{HAL Id: hal-00687408 https://hal.science/hal-00687408}

Submitted on 13 Apr 2012

HAL is a multi-disciplinary open access archive for the deposit and dissemination of scientific research documents, whether they are published or not. The documents may come from teaching and research institutions in France or abroad, or from public or private research centers.
L'archive ouverte pluridisciplinaire HAL, est destinée au dépôt et à la diffusion de documents scientifiques de niveau recherche, publiés ou non, émanant des établissements d'enseignement et de recherche français ou étrangers, des laboratoires publics ou privés. 


\title{
Separation of Sparse Signals in Overdetermined Linear-Quadratic Mixtures
}

\author{
Leonardo T. Duarte ${ }^{1}$, Rafael A. Ando ${ }^{2, \star}$, Romis Attux ${ }^{2}$, \\ Yannick Deville ${ }^{3}$, and Christian Jutten ${ }^{4}$ \\ 1 School of Applied Sciences - University of Campinas (UNICAMP), \\ Campinas, Brazil \\ leonardo.duarte@fca.unicamp.br \\ 2 School of Electrical and Computer Engineering - University of Campinas \\ (UNICAMP), Campinas, Brazil \\ \{assato, attux\}@dca.fee.unicamp.br \\ 3 IRAP, Université de Toulouse, CNRS, Toulouse, France \\ ydeville@ast.obs-mip.fr \\ 4 GIPSA-Lab, CNRS UMR-5216, Grenoble, and Institut Universitaire de France \\ christian.jutten@gipsa-lab.grenoble-inp.fr
}

\begin{abstract}
In this work, we deal with the problem of nonlinear blind source separation (BSS). We propose a new method for BSS in overdetermined linear-quadratic (LQ) mixtures. By exploiting the assumption that the sources are sparse in a transformed domain, we define a framework for canceling the nonlinear part of the mixing process. After that, separation can be conducted by linear BSS algorithms. Experiments with synthetic data are performed to assess the viability of our proposal.
\end{abstract}

Keywords: Nonlinear mixtures, sparse signals, blind source separation.

\section{Introduction}

In blind source separation (BSS), the goal is to retrieve a set of signals (sources) based only on the observation of mixed versions of these original sources 112. Typically, the methods developed to solve this problem work with the assumption that the mixing process can be modeled as a linear system. However, while this framework has been proven successful in many applications, there are some practical examples in which the mixtures are clearly nonlinear - this is the case, for instance, in chemical sensor arrays $[3$ and hyperspectral imaging 4 .

Several works have already pointed out some problems that arise when the mixtures are nonlinear (see [5] for a discussion). In particular, the application of methods based on independent component analysis (ICA) [6], which assumes that the sources are mutually statistically independent random variables, is not valid in a general nonlinear system. In view of this problem, the research on nonlinear BSS has been focused on constrained models, for which the task of

\footnotetext{
${ }^{\star}$ R. Assato would like to thank FAPESP (Brazil) for funding his MSc studies.
} 
source separation can be accomplished by extending the ideas already considered in the linear case. Among the constrained nonlinear models studied so far is the linear-quadratic (LQ) model [7/4. This model provides a good description of the mixing process in applications such as show-through effect removal in scanned images [8] and design of gas sensor arrays [9]. Besides, the LQ model presents two interesting properties: 1) it can be seen as a first step toward more general polynomial mixtures; 2) it is linear with respect to the mixing coefficients.

When the number of sources is equal to the number of mixtures in an LQ model, the definition of a separating structure is not a simple task, given the difficulty in writing the inverse of the mixing process in an analytical form [7]10]. However, in an overdetermined case, in which the number of mixtures is greater than the number of sources, separation can be achieved by means of a linear structure. This idea has already been exploited in the context of sources belonging to a finite alphabet [11, circular sources [12], non-stationary sources [13] and independent sources 14 .

In the present work, we tackle the problem of BSS in overdetermined mixtures assuming that the sources admit a sparse representation in a given basis. By using this property, we propose a strategy to cancel the nonlinear part of the LQ mixing process, so that the resulting problem can be dealt with by linear BSS algorithms. Since our approach for dealing with the nonlinear terms does not rely on the independence assumption, it is possible to tackle problems in which ICA methods fail. Of course, this can be done if the adopted linear BSS method is able to work with dependent mixtures.

\section{Mixing Model}

Let $\mathbf{s}_{j}=\left[s_{j}(1) \ldots s_{j}\left(n_{d}\right)\right]^{T}$ represent $j$-th source $\left(n_{d}\right.$ is the number of samples). In the present work, we consider the case of $n_{s}=2$ sources, which is representative in applications such as the design of gas sensor arrays and separation of scanned mixtures. In this case, the $i$-th mixture can be represented by the vector

$$
\mathbf{x}_{i}=a_{i 1} \mathbf{s}_{1}+a_{i 2} \mathbf{s}_{2}+a_{i 3} \mathbf{s}_{1} \circ \mathbf{s}_{2},
$$

where $\circ$ stands for the element-wise product operator (Hadamard product), while the mixing coefficients are denoted by $a_{i j}$.

An interesting aspect of (1) is that it can be interpreted as a linear mixing process in which the sources are given by $\mathbf{s}_{1}, \mathbf{s}_{2}$ and $\mathbf{s}_{1} \circ \mathbf{s}_{2}$. Therefore, in an overdetermined case, with three mixtures given by

$$
\left[\begin{array}{l}
x_{1}(n) \\
x_{2}(n) \\
x_{3}(n)
\end{array}\right]=\left[\begin{array}{lll}
a_{11} & a_{12} & a_{13} \\
a_{21} & a_{22} & a_{23} \\
a_{31} & a_{32} & a_{33}
\end{array}\right]\left[\begin{array}{c}
s_{1}(n) \\
s_{2}(n) \\
s_{1}(n) s_{2}(n)
\end{array}\right], \forall n \in\left\{1, \ldots, n_{d}\right\},
$$

it is possible to achieve source separation by means of a linear separating system, in which the recovered sources are given by

$$
\left[\begin{array}{l}
y_{1}(n) \\
y_{2}(n)
\end{array}\right]=\left[\begin{array}{lll}
w_{11} & w_{12} & w_{13} \\
w_{21} & w_{22} & w_{23}
\end{array}\right]\left[\begin{array}{l}
x_{1}(n) \\
x_{2}(n) \\
x_{3}(n)
\end{array}\right], \forall n \in\left\{1, \ldots, n_{d}\right\}
$$


As will be discussed in the sequel, source separation in this case can be performed by firstly canceling the nonlinear elements of the mixtures, followed by the application of a linear BSS method.

\section{Sparsity-Based Cancellation of Quadratic Terms}

\subsection{The Main Idea}

Since we have access to, at least, three mixtures, it is possible to linearly combine them in order to extract the quadratic term expressed in (2). Let us consider the linear combination between the mixtures $\mathbf{x}_{i}$ and $\mathbf{x}_{j}$ :

$$
\mathbf{z}_{i j}=\mathbf{x}_{i}-\alpha_{i j} \mathbf{x}_{j}
$$

where the index $i j$ corresponds to the mixtures considered in the combination. According to (1), $\mathbf{z}_{i j}$ can be rewritten as follows

$$
\mathbf{z}_{i j}=\left(a_{i 1}-\alpha_{i j} a_{j 1}\right) \mathbf{s}_{1}+\left(a_{i 2}-\alpha_{i j} a_{j 2}\right) \mathbf{s}_{2}+\left(a_{i 3}-\alpha_{i j} a_{j 3}\right) \mathbf{s}_{1} \circ \mathbf{s}_{2}
$$

Therefore, when $\alpha_{i j}=a_{i 3} / a_{j 3}, \mathbf{z}_{i j}$ becomes a linear mixture of $\mathbf{s}_{1}$ and $\mathbf{s}_{2}$.

The implementation of the idea described above requires the definition of a criterion to guide the estimation of $\alpha_{i j}$. A possible idea to accomplish this task can be formulated by rewriting (5) in a transformed domain, which is achieved by multiplying $\mathbf{z}_{i j}$ by the $n_{d} \times n_{d}$ orthonormal matrix $\boldsymbol{\Phi}$ that represents such a transformation. In mathematical terms,

$$
\begin{aligned}
\mathbf{z}_{i j}^{\prime}=\boldsymbol{\Phi} \mathbf{z}_{i j} & =\left(a_{i 1}-\alpha_{i j} a_{j 1}\right) \boldsymbol{\Phi} \mathbf{s}_{1}+\left(a_{i 2}-\alpha_{i j} a_{j 2}\right) \boldsymbol{\Phi} \mathbf{s}_{2}+\left(a_{i 3}-\alpha_{i j} a_{j 3}\right) \boldsymbol{\Phi}\left(\mathbf{s}_{1} \circ \mathbf{s}_{2}\right) \\
& =\left(a_{i 1}-\alpha_{i j} a_{j 1}\right) \mathbf{s}_{1}^{\prime}+\left(a_{i 2}-\alpha_{i j} a_{j 2}\right) \mathbf{s}_{2}^{\prime}+\left(a_{i 3}-\alpha_{i j} a_{j 3}\right) \boldsymbol{\Phi}\left(\mathbf{s}_{1} \circ \mathbf{s}_{2}\right),
\end{aligned}
$$

where $\mathbf{s}_{i}^{\prime}$ is the representation in the transformed domain of the source $\mathbf{s}_{i}$.

Let us consider for instance that the orthonormal matrix $\boldsymbol{\Phi}$ is related to a frequency transformation, e.g. the discrete cosine transform (DCT). Moreover, let us assume that both $\mathbf{s}_{1}^{\prime}$ and $\mathbf{s}_{2}^{\prime}$ are sparse vectors, in the sense that not all frequency components of these signals are not null. Note that the term $\boldsymbol{\Phi}\left(\mathbf{s}_{1} \circ \mathbf{s}_{2}\right)$ in (6) is related to the convolution of $\mathbf{s}_{1}^{\prime}$ and $\mathbf{s}_{2}^{\prime}$, since it is given by the DCT transform of a product in time. The key point here is that the convolution of $\mathbf{s}_{1}^{\prime}$ and $\mathbf{s}_{2}^{\prime}$ tends to produce a signal that is not sparse, or at least less sparse than $\mathbf{s}_{1}^{\prime}$ and $\mathbf{s}_{2}^{\prime}$, as the nonlinear term causes a spreading in the frequency domain - this feature is illustrated in Figure 11. Based on this observation, our idea is to adjust $\alpha_{i j}$ by maximizing the degree of sparsity of $\mathbf{z}_{i j}^{\prime}$. If the $\ell_{0}$-norm, which corresponds to the number of non-zero elements of a vector, is adopted as a measure of sparsity [15, then our idea can be formulated as the following optimization problem

$$
\min _{\alpha_{i j}}\left\|\boldsymbol{\Phi} \mathbf{z}_{i j}\right\|_{0}
$$

It is worth mentioning that the matrix $\boldsymbol{\Phi}$ should not necessarily be related with a frequency transform. The only requirement is that $\boldsymbol{\Phi}$ somehow spreads the representation of $\left(\mathbf{s}_{1} \circ \mathbf{s}_{2}\right)$. This point will be further discussed in the sequel. 


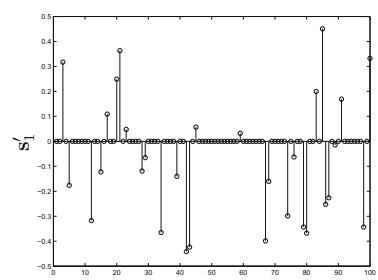

(a) $\mathbf{s}_{1}^{\prime}$.

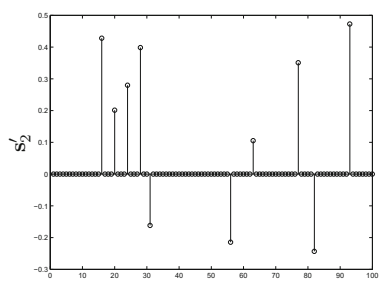

(b) $\mathbf{s}_{2}^{\prime}$.

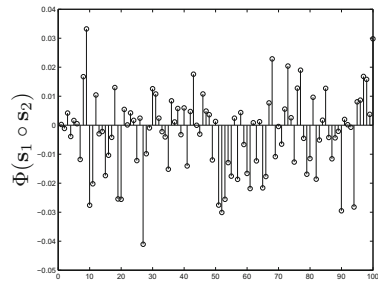

(c) $\boldsymbol{\Phi}\left(\mathbf{s}_{1} \circ \mathbf{s}_{2}\right)$.

Fig. 1. DCTs of the sources and of the product between these sources

\subsection{Theoretical Aspects}

We here discuss some theoretical aspects related to the idea expressed in (7). In particular, we provide the guidelines for establishing general conditions for which our proposal is valid. In our analysis, we assume that the mixing matrix is full rank. Moreover, we assume that $\left\|\boldsymbol{\Phi}\left(\mathbf{s}_{1} \circ \mathbf{s}_{2}\right)\right\|_{0} \geq \max \left(\left\|\mathbf{s}_{1}^{\prime}\right\|_{0},\left\|\mathbf{s}_{2}^{\prime}\right\|_{0}\right)$. As our analysis is based on the $\ell_{0}$-norm, it is important to introduce some properties of this measure, which strictly speaking is not a mathematical norm [15. Yet, the $\ell_{0}$-norm satisfies the triangle inequality, that is, given two vectors $\mathbf{a}$ and $\mathbf{b}$, then $\|\mathbf{a}+\mathbf{b}\|_{0} \leq\|\mathbf{a}\|_{0}+\|\mathbf{b}\|_{0}$. As a consequence, the $\ell_{0}$-norm also satisfies the reverse triangle inequality, i.e., $\|\mathbf{a}-\mathbf{b}\|_{0} \geq\left|\|\mathbf{a}\|_{0}-\|\mathbf{b}\|_{0}\right|$. Finally, the $\ell_{0}$-norm is scale invariant, i.e., $\|k \mathbf{a}\|_{0}=\|\mathbf{a}\|_{0}$ for $k \neq 0$.

In order to investigate the cost function $\left\|\boldsymbol{\Phi} \mathbf{z}_{i j}\right\|_{0}$, let us rewrite (6) as follows:

$$
\mathbf{z}_{i j}^{\prime}=a \mathbf{s}_{1}^{\prime}+b \mathbf{s}_{2}^{\prime}+c \boldsymbol{\Phi}\left(\mathbf{s}_{1} \circ \mathbf{s}_{2}\right) .
$$

Ideally, to be in accordance with our idea, $\left\|\mathbf{z}_{i j}^{\prime}\right\|_{0}$ should attain a minimum if, and only if, $\alpha_{i j}=a_{i 3} / a_{j 3}$, that is, when $c=0$. In this case, one only has the linear terms of the mixture, and, thus, by considering the triangle inequality and the scaling invariance property, it turns out that

$$
\left\|\mathbf{z}_{i j}^{\prime}\right\|_{0} \leq\left\|\mathbf{s}_{1}^{\prime}\right\|_{0}+\left\|\mathbf{s}_{2}^{\prime}\right\|_{0} .
$$

We can also investigate $\left\|\mathbf{z}_{i j}^{\prime}\right\|_{0}$ in the cases in which $\alpha_{i j}$ does not lead to the cancellation of the quadratic term, i.e. when $c \neq 0$ in (8). In these situations, our idea will work when $\left\|\mathbf{z}_{i j}^{\prime}\right\|_{0}$ is greater than the upper bound (9). When $a=0$, $b \neq 0$, and $c \neq 0$, one can use the reverse triangle inequality to obtain the following lower bound

$$
\left\|\mathbf{z}_{i j}^{\prime}\right\|_{0}=\left\|b \mathbf{s}_{2}^{\prime}+c \boldsymbol{\Phi}\left(\mathbf{s}_{1} \circ \mathbf{s}_{2}\right)\right\|_{0} \geq\left\|\boldsymbol{\Phi}\left(\mathbf{s}_{1} \circ \mathbf{s}_{2}\right)\right\|_{0}-\left\|\mathbf{s}_{2}^{\prime}\right\|_{0} .
$$

Analogously, when $a \neq 0, b=0$, and $c \neq 0$, one can easily show that

$$
\left\|\mathbf{z}_{i j}^{\prime}\right\|_{0} \geq\left\|\boldsymbol{\Phi}\left(\mathbf{s}_{1} \circ \mathbf{s}_{2}\right)\right\|_{0}-\left\|\mathbf{s}_{1}^{\prime}\right\|_{0} .
$$


Finally, when $a \neq 0, b \neq 0$, and $c \neq 0$, the following lower bound for $\left\|\mathbf{z}_{i j}^{\prime}\right\|_{0}$ can be obtained after the application of the triangle inequality followed by the application of the reversed triangle inequality

$$
\left\|\mathbf{z}_{i j}^{\prime}\right\|_{0} \geq\left\|\boldsymbol{\Phi}\left(\mathbf{s}_{1} \circ \mathbf{s}_{2}\right)\right\|_{0}-\left\|\mathbf{s}_{1}^{\prime}\right\|_{0}-\left\|\mathbf{s}_{2}^{\prime}\right\|_{0} .
$$

Among the bounds expressed in (10), (11) and (12), and the bound $\left\|\boldsymbol{\Phi}\left(\mathbf{s}_{1} \circ \mathbf{s}_{2}\right)\right\|_{0}$ obtained when $a=0, b=0, c \neq 0$, the bound shown in (12) is the smallest one. Therefore, if the lower bound (12) is greater than the higher bound (9), then $\left\|\boldsymbol{\Phi} \mathbf{z}_{i j}\right\|_{0}$ will necessarily reach the global minimum at $\alpha_{i j}=a_{i 3} / a_{j 3}($ i.e. $c=0)$. This observation leads to the following sufficient condition:

$$
\left\|\boldsymbol{\Phi}\left(\mathbf{s}_{1} \circ \mathbf{s}_{2}\right)\right\|_{0}-\left\|\mathbf{s}_{1}^{\prime}\right\|_{0}-\left\|\mathbf{s}_{2}^{\prime}\right\|_{0}>\left\|\mathbf{s}_{1}^{\prime}\right\|_{0}+\left\|\mathbf{s}_{2}^{\prime}\right\|_{0}
$$

i.e.

$$
\left\|\boldsymbol{\Phi}\left(\mathbf{s}_{1} \circ \mathbf{s}_{2}\right)\right\|_{0}>2\left(\left\|\mathbf{s}_{1}^{\prime}\right\|_{0}+\left\|\mathbf{s}_{2}^{\prime}\right\|_{0}\right)
$$

Some observations can be made on this condition. Firstly, the importance of defining a proper transformation, for which the representation of the quadratic terms is as spread as possible, becomes clear. Note that such a requirement becomes less stringent when the sources have a high degree of sparsity in the transformed domain. For instance, in the situation illustrated in Figure 1, it is clear that the DCT satisfies the sufficient condition expressed in (14). Moreover, when $\boldsymbol{\Phi}$ is given by a random matrix submitted to an orthogonalization process, condition (14) can be satisfied for many different configurations of the sources.

A second point related to (14) is that it provides a sufficient but not necessary condition. Actually, this condition is quite pessimistic, as it considers a very peculiar configuration of the positions for which the signals $\mathbf{s}_{1}^{\prime}$ and $\mathbf{s}_{2}^{\prime}$ take nonzero values.

\subsection{Implementation Issues}

In a practical application, the use of the $\ell_{0}$-norm is quite limited, since sparse signals in practice have many elements that are close to zero, but that are not necessarily null. Thus, approximations of the $\ell_{0}$-norm must be considered. A possible choice is the smoothed version of $\ell_{0}$-norm [16], which, for a given signal $\mathbf{y}$, is defined as follows:

$$
S_{\ell_{0}}(\mathbf{y})=n_{d}-\sum_{i=1}^{n_{d}} f\left(y_{(i)}, \sigma\right),
$$

where $f(\cdot, \sigma)$ corresponds to a zero mean Gaussian kernel of standard deviation $\sigma$. As $\sigma$ approaches to zero, (15) approaches to the $\ell_{0}$-norm. Ideally, the choice of $\sigma$ depends on how close to zero the low-energy elements of a given signal are.

We can now introduce a general scheme for source separation in overdetermined LQ mixtures of two sources. The proposal, which can be applied when the number of mixtures is greater than 2 , is composed of the following steps: 1 ) Cancellation of quadratic terms: considering $n_{p}>1$ pairs of mixtures, $\mathbf{x}_{i}$ and 
$\mathbf{x}_{j}$, find, for each of these pairs, an $\alpha_{i j}$ which minimizes $S_{\ell_{0}}\left(\mathbf{z}_{i j}^{\prime}\right)$. This procedure will provide the set of signals that, ideally, correspond to linear mixtures of the sources. 2) Source separation: apply a linear source separation (or extraction) method on the signals obtained in the first stage.

The first stage of the proposed strategy boils down to $n_{p}$ univariate optimization problems, which, in our work, are carried out by an exhaustive search approach. Note that the first stage can be conducted even when the sources are not statistically independent. Of course, in this case, the second stage should be able to deal with linear mixtures of dependent sources.

\section{Results}

Let us consider an overdetermined LQ source separation problem in which the

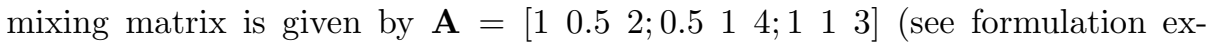
pressed in (2) ). The sources here are sparse in the DCT domain. To generate the DCT coefficients, we firstly obtained 500 samples from a distribution uniformly distributed in $[-0.5,0.5]$. Then, we replaced a given percentage of the generated elements by samples obtained from a zero-mean Gaussian distribution of standard deviation 0.001 (these are the low-energy DCT coefficients). The percentages of these low-energy elements were $90 \%$ for the first source and $70 \%$ for the second source, and their position were randomly selected. Finally, the sources shared 50 DCT coefficients (these coefficiecients are not the ones with small values), which make them statistically dependent.

In order to remove the quadratic terms in the mixtures, we applied the proposed method to the pairs of mixtures $\left(\mathbf{x}_{1}, \mathbf{x}_{2}\right)$ and $\left(\mathbf{x}_{1}, \mathbf{x}_{3}\right)$. After performing 10 runs, each one considering a different set of sources generated according to the procedure described above, our method provided very good solutions in every runs. Indeed, the obtained mean values were $\alpha_{12}=0.5012$ and $\alpha_{13}=0.6671$, which are very close to the ideal values $\alpha_{12}=1 / 2$ and $\alpha_{13}=2 / 3$. To illustrate that the nonlinear terms were removed in this situation, we plot in Figure 2 the DCT coefficients of the sources, mixtures and the provided signals $\mathbf{z}_{12}$ and $\mathbf{z}_{13}$ obtained in a given run. Note that the DCT coefficients of the obtained signals are clearly sparser than those of the mixtures.

With the obtained linear mixtures at hand, we applied the source extraction method proposed in [17] to retrieve the sparsest component. As discussed in [17, this method is able to conduct source separation even when the sources are dependent. Indeed, the sparsest source was estimated with a signal-to-interference ratid 1 (SIR) of $58.7 \mathrm{~dB}$. On the other hand, the SIRs obtained after the application of the ICA-based solution proposed in 14 were $4.0 \mathrm{~dB}$ (first source) and $7.1 \mathrm{~dB}$ (second source). These low values can be attributed to the fact that the sources were not independent in the considered scenario, thus violating the central assumption of ICA methods.

${ }^{1}$ The SIR is defined as $=10 \log \left(E\left\{\hat{y}_{i}^{2}\right\} / E\left\{\left(\hat{s}_{i}-\hat{y}_{i}\right)^{2}\right\}\right)$, where $\hat{s}_{i}$ and $\hat{y}_{i}$ are, respectively, the actual source and its estimate, being both ones obtained after mean, variance and sign normalization. 


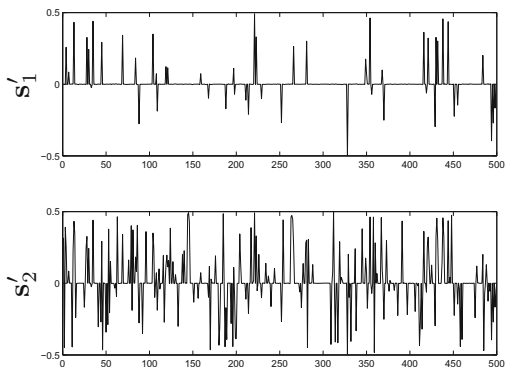

(a) Sources.

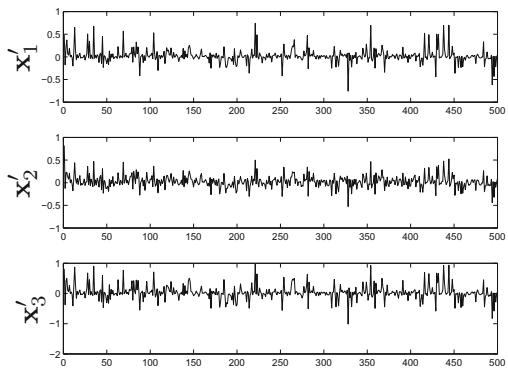

(b) Mixtures.
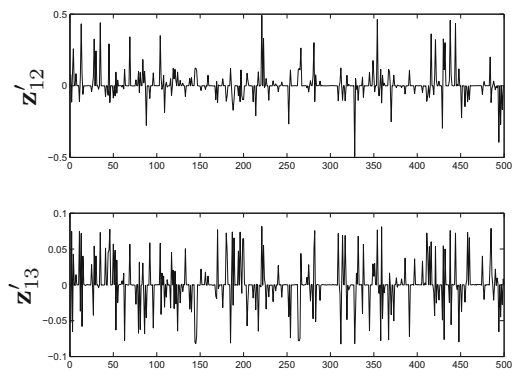

(c) $\boldsymbol{\Phi}\left(\mathbf{s}_{1} \circ \mathbf{s}_{2}\right)$.

Fig. 2. Obtained linear mixtures

\section{Conclusions}

We proposed a method for suppressing the quadratic terms of overdetermined LQ mixtures. Our approach works with the assumption that the sources are sparse when represented in a proper domain, which should be known in advance, and is based on a $\ell_{0}$-norm minimization procedure. We provided theoretical elements that points out that our proposal is suitable for the cases in which the quadratic terms admit a representation in the considered domain that is less sparse than those of the sources. A numerical experiment illustrated the effectiveness of the obtained method, especially when the sources are dependent.

There are several points to be investigated in future works. For instance, a first one is to extend the theoretical analysis conducted in this paper to the case of the smoothed $\ell_{0}$-norm, paying special attention to the influence of the parameter $\sigma$. Another relevant point is to investigate if the two-stage procedure described in Section 3.3 can be merged into a unique step guided by the minimization of the sparsity of the retrieved sources. Finally, we intent to investigate the extension of the idea to the case in which the number of sources is greater than two, and its application to actual problems. 


\section{References}

1. Comon, P., Jutten, C. (eds.): Handbook of blind source separation, independent component analysis and applications. Academic Press, Elsevier (2010)

2. Romano, J.M.T., Attux, R.R.F., Cavalcante, C.C., Suyama, R.: Unsupervised signal processing: channel equalization and source separation. CRC Press (2011)

3. Duarte, L.T., Jutten, C., Moussaoui, S.: A Bayesian nonlinear source separation method for smart ion-selective electrode arrays. IEEE Sensors Journal 9(12), 1763-1771 (2009)

4. Meganem, I., Deville, Y., Hosseini, S., Déliot, P., Briottet, X., Duarte, L.T.: Linearquadratic and polynomial non-negative matrix factorization; application to spectral unmixing. In: Proc. of the 19th European Signal Processing Conference, EUSIPCO 2011 (2011)

5. Jutten, C., Karhunen, J.: Advances in blind source separation (BSS) and independent component analysis (ICA) for nonlinear mixtures. International Journal of Neural Systems 14, 267-292 (2004)

6. Comon, P.: Independent component analysis, a new concept? Signal Processing 36, 287-314 (1994)

7. Hosseini, S., Deville, Y.: Blind Separation of Linear-Quadratic Mixtures of Real Sources Using a Recurrent Structure. In: Mira, J., Álvarez, J.R. (eds.) IWANN 2003. LNCS, vol. 2687, pp. 241-248. Springer, Heidelberg (2003)

8. Merrikh-Bayat, F., Babaie-Zadeh, M., Jutten, C.: Linear-quadratic blind source separating structure for removing show-through in scanned documents. International Journal on Document Analysis and Recognition, 1-15 (2010)

9. Bedoya, G.: Nonlinear blind signal separation for chemical solid-state sensor arrays. $\mathrm{PhD}$ thesis, Universitat Politecnica de Catalunya (2006)

10. Deville, Y., Hosseini, S.: Recurrent networks for separating extractable-target nonlinear mixtures. part i: Non-blind configurations. Signal Processing 89, 378-393 (2009)

11. Castella, M.: Inversion of polynomial systems and separation of nonlinear mixtures of finite-alphabet sources. IEEE Trans. on Sig. Proc. 56(8), 3905-3917 (2008)

12. Abed-Meraim, K., Belouchrani, A., Hua, Y.: Blind identification of a linearquadratic mixture of independent components based on joint diagonalization procedure. In: Proc. of the IEEE Inter. Conf. on Acous., Spee., and Signal Processing, ICASSP (1996)

13. Deville, Y., Hosseini, S.: Blind identification and separation methods for linearquadratic mixtures and/or linearly independent non-stationary signals. In: Proc. of the 9th Int. Symp. on Sig. Proc. and its App., ISSPA (2007)

14. Duarte, L.T., Suyama, R., Attux, R., Deville, Y., Romano, J.M.T., Jutten, C.: Blind Source Separation of Overdetermined Linear-Quadratic Mixtures. In: Vigneron, V., Zarzoso, V., Moreau, E., Gribonval, R., Vincent, E. (eds.) LVA/ICA 2010. LNCS, vol. 6365, pp. 263-270. Springer, Heidelberg (2010)

15. Elad, M.: Sparse and redundant representations from theory to applications in signal and image processing. Springer, Heidelberg (2010)

16. Mohimani, H., Babaie-Zadeh, M., Jutten, C.: A fast approach for overcomplete sparse decomposition based on smoothed $\ell^{0}$ norm. IEEE Transactions on Signal Processing 57(1), 289-301 (2009)

17. Duarte, L.T., Suyama, R., Attux, R., Romano, J.M.T., Jutten, C.: Blind extraction of sparse components based on $\ell_{0}$-norm minimization. In: Proc. of the IEEE Statistical Signal Processing Workshop, SSP (2011) 